\title{
Doença de madelung e carcinoma hepatocelular: relato de caso
}

\author{
Madelung's disease and hepatocellular carcinoma: case report
}

Cid C.S. Alcântara ${ }^{1}$, Christiane A. Nobre'2, Mirella M. B. Monteiro³, Lara R. S. Freitas ${ }^{4}$

\begin{abstract}
RESUMO
A doença de Madelung, lipomatose cervical benigna simétrica, é afecção rara, de etiologia desconhecida caracterizada por acúmulo de volumosas massas de gordura não encapsuladas. Existem poucos estudos sobre a doença de Madelung e associação com neoplasias. Descrevemos um caso de doença de Madelung associada a carcinoma hepatocelular.
\end{abstract}

Palavras-chave: Doença de Madelung. Alcoolismo. Carcinoma Hepatocelular. Lipomatose Adenomatosa.

\section{Introdução}

A doença de Madelung, lipomatose cervical benigna simétrica, é afecção rara, de etiologia desconhecida. ${ }^{1}$ Foi mencionada na literatura pela primeira vez por Brodie em 1846, e uma série de 33 casos foi descrita por Madelung em 1888. Dez anos mais tarde, Launois-Bensaude descreveu 65 casos semelhantes, que foram chamados de adenolipomatose múltipla simétrica ${ }^{2}$. Acomete mais frequentemente indivíduos do sexo masculino, estando associada frequentemente ao etilismo ${ }^{2}$. Existem poucos estudos sobre a doença de Madelung e associação com neoplasias. O objetivo deste estudo foi relatar um caso de doença de Madelung associada a carcinoma hepatocelular.

\section{Relato do caso}

Paciente masculino, 62 anos, há 10 anos com crescimento progressivo de massas indolores em região lombar e nádegas que evoluíram para dorso, região cervical e parte proximal de membros superiores. Etilista diário e tabagista há mais de 30 anos. Biópsia das lesões compatível com lipoma sendo diagnosticada Doença de Madelung e submetido à lipectomia cervical e dorsal. Apresentou recidiva das lesões e ganho ponderal de $40 \mathrm{Kg}$ ao longo de 10 anos.

Procurou atendimento médico por surgimento de cefaleia holocraniana. Ao exame físico destacavase a presença de nódulo endurecido em região frontotemporal direita bem como múltiplos nódulos com apro-
1. Residente de Clínica Médica da Universidade Federal do Ceará. Mestrado em Saúde Pública pela Universidade Federal do Ceará

2. Professora do Curso de Medicina da Universidade Federal do Ceará. Mestrado em Biotecnologia pela Universidade Federal do Ceará

3. Residência em Medicina de Família e Comunidade e especialista em Medicina estética e Homeopatia

4. Professora auxiliar da Universidade de Fortaleza
Correspondência Cid Carlos Soares de Alcântara R. Três número 88 - Dias Macedo 60860-470 Fortaleza, CE. E-mail: cidcsa@gmail.com

Os autores declaram não haver conflito de interesses

Artigo recebido em 18/04/2013 Aprovado para publicação em 29/11/2013 
ximadamente $10 \mathrm{~cm}$ no seu maior diâmetro, com consistência amolecida, móveis, presentes em região cervical posterior, proximal de membros superiores e glútea. Foi internado para esclarecimento diagnóstico. Os nódulos frontais foram biopsiados e submetidos a imunohistoquímica. Os resultados dos exames de maior relevância para a investigação diagnóstica encontram-se na Tabela I. Sendo confirmada a presença de nódulos cranianos metastáticos provenientes de hepatocarcinoma.

Submetido a radioterapia com diminuição das lesões, sendo encaminhado para acompanhamento ambulatorial.

Após quatro meses, evoluiu com intensa dor óssea generalizada, disfagia para líquidos e perda de

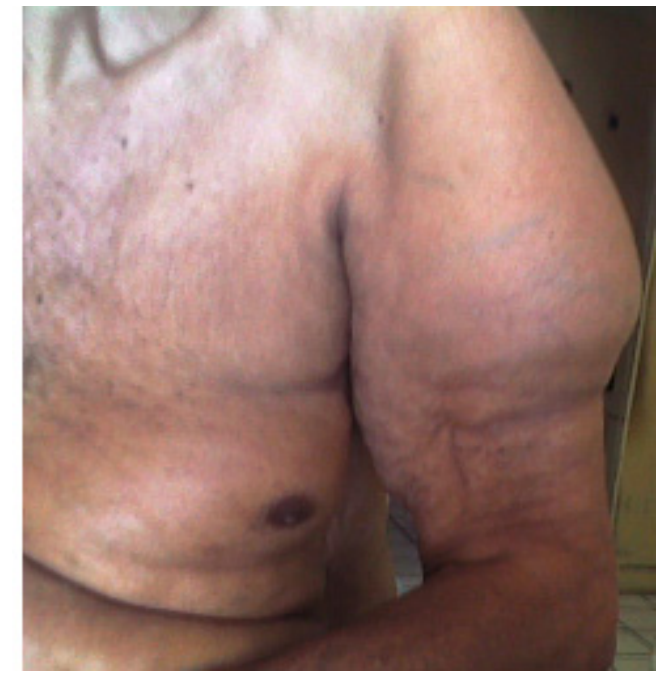

Figura 1. Gordura subcutânea acumulada na região do deltoide dando aparência pseudoatlética peso sendo necessário novo internamento. Em 20 dias evoluiu com comprometimento do estado geral, pneumonia grave e insuficiência respiratória, evoluindo para óbito.

\section{Discussão}

O diagnóstico da Doença de Madelung é feito através da história clínica e exame físico. O diagnóstico diferencial inclui doenças como neurofibromas, angiolipomas, lipomatose familiar múltipla, sarcomas, lipoblastomas, síndrome de Dercum, síndrome de Hanhart, síndrome da polidisplasia, doença de Cushing, doenças linfoproliferativas, doenças da tireoide e distrofias musculares ${ }^{3,4}$. A biópsia é fundamental para

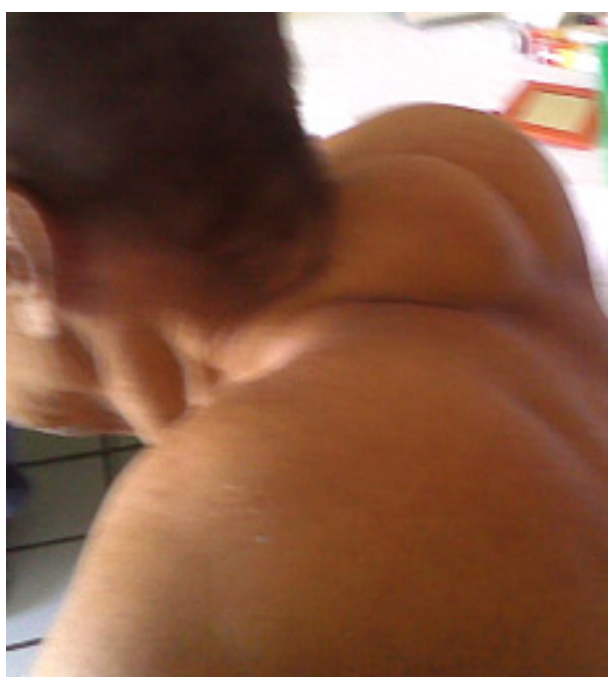

Figura 2. Gordura na região cervical posterior semelhante a uma giba

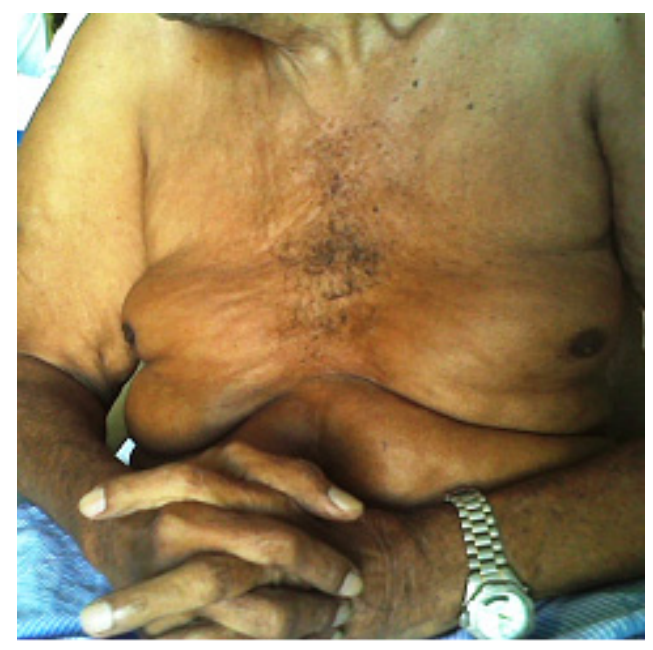

Figura 3. Acúmulo de gordura em região do peito e abdômen 


\section{Tabela I}

Exames realizados durante o internamento

\begin{tabular}{llc}
\hline Exames & Resultados & Valores de referência \\
\hline Alfa-feto-proteína & $4,1 \mathrm{mcg} / \mathrm{L}$ & $\leq 15 \mathrm{mcg} / \mathrm{L}$ \\
Beta-2-microglobulina & $3,4 \mathrm{mg} / \mathrm{dl}$ & $\leq 0,27 \mathrm{mcg} / \mathrm{ml}$ \\
HBs-Ag, Anti-HBc & Negativos & \\
Anti-HCV & Negativo & \\
$\mathrm{Ca} 19,9$ & $<3,0 \mathrm{U} / \mathrm{ml}$ (normal) & $\leq 37 \mathrm{U} / \mathrm{ml}$ \\
PSA & $1,2 \mathrm{ng} / \mathrm{ml}$ (normal) & $\leq 4 \mathrm{ng} / \mathrm{ml}$ \\
Laringoscopia indireta & Normal & \\
Endoscopia Digestiva Alta & Gastrite enantematosa leve & \\
Tomografia Abdome & Imagens nodulares hepáticas em lobo direito, algumas & \\
& coalescentes nos segmentos V e VI & \\
Tomografia de Crânio & Lesões expansivas extra-axiais com aspecto sugestivo & \\
& de implantes neoplásicos secundários com pequenas & \\
Biópsia de nódulo da região glútea & Lipoma de infartos lacunares. & \\
Biópsia de nódulo da região & Metástase de carcinoma hepato-celular bem diferenciado \\
frontal & trabecular \\
Imunohistoquímica do nódulo da & Compatível com hepatocarcinoma metastático & \\
região frontal & & \\
\hline
\end{tabular}

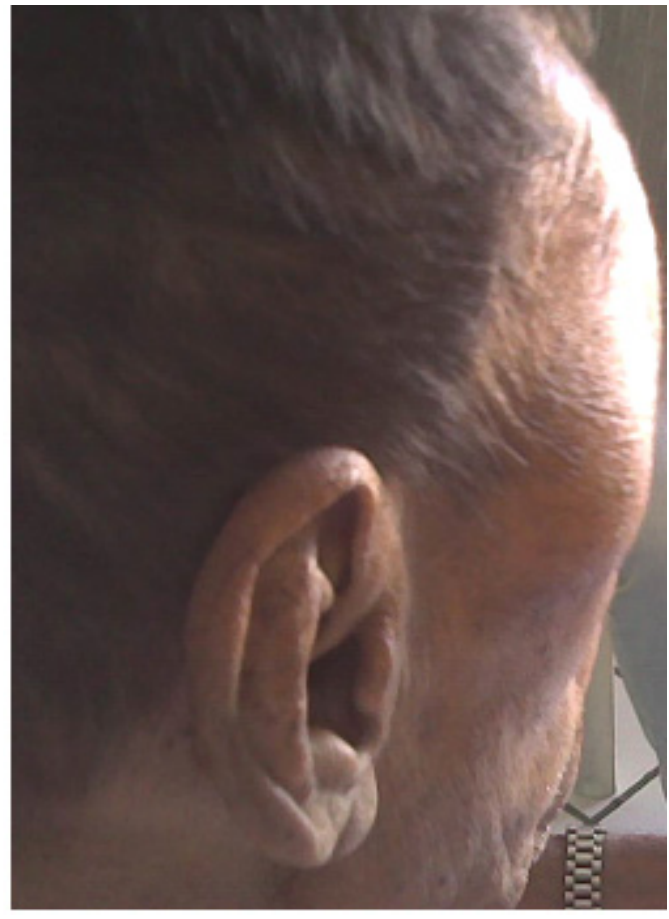

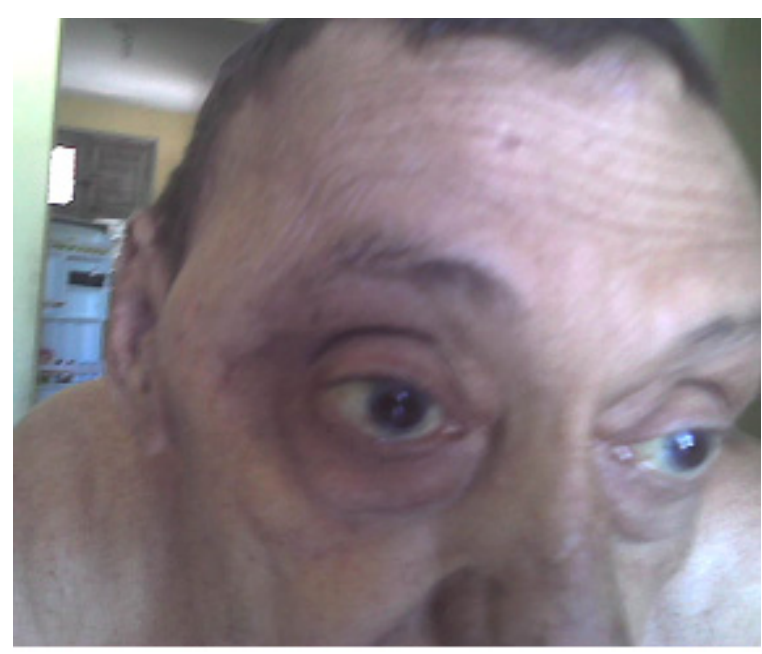

Figura 5. Metástase óssea em região temporal direita

Figura 4. Metástase óssea em região temporal direita 
se excluir eventuais malignidades. Os exames de avaliação radiológica solicitados como a tomografia computadorizada e/ou a ressonância nuclear magnética podem visualizar lipomatose, calcificação e ossificação dentro dos lipomas. Em um estudo longitudinal que acompanhou pacientes com doença de Madelung, os sintomas associados em ordem decrescente de prevalência foram parestesias, câimbras, roncos noturnos, dispneia, disfagia, sudorese, taquicardia, hipersonia e disfunção erétil ${ }^{5}$.

A doença é classificada em tipo 1: os depósitos de gordura são circunscritos, formando massas nãoencapsuladas distribuídas simetricamente na porção superior do corpo. Formam-se lipomas nas regiões cervical (colar de cavalo), deltóidea (aparência pseudoatlética) e na parte superior das costas dando a falsa impressão que o paciente apresenta cifose de coluna vertebral. O tipo 2 afeta homens e mulheres, produzindo uma exagerada distribuição feminina de gordura na região superior das coxas, quadris, braços e região superior das costas ${ }^{6}$.

A lipomatose é associada com hipertensão arterial, anemia macrocítica, dano hepático associado ao álcool, intolerância a glicose, diabetes mellitus, hiperlipoproteinemia, hipertrigliceridemia, colesterol total e HDL normal ou aumentado, hiperuricemia, acidose tubular renal, tumores malignos das vias aéreas superiores, esteatose hepática, cirrose, polineuropatia periférica ${ }^{7}$.

A persistência do consumo de etanol acelera o crescimento do tecido lipomatoso ao mesmo tempo em que a interrupção de álcool está associado uma ligeira regressão dos depósitos lipomatosos $\mathrm{O}$ etanol parece agir como um gatilho da formação e crescimento da lipomatose. Abstinência de álcool é uma medida não cirúrgica a ser implementada durante o acompanhamento destes pacientes ${ }^{5}$.

A doença de Madelung é uma doença com associação com neoplasias. Existem estudos de associação com neoplasia lipomatosa das glândulas salivares, adenocarcinoma de esôfago, câncer de mama, pulmão, intestino e transformação neoplásica dos lipomas ${ }^{7,8,9,10}$. Esse é o primeiro caso descrito na literatura dessa doença associado à hepatocarcinoma.

Enzi G et al, 2002 realizaram estudo que acompanhou 31 pacientes portadores de doença de Madelung. Dos oito pacientes (25,8\%) que evoluíram para o óbito, três $(37,5 \%)$ tiveram óbito por neoplasias. Apesar da elevada ingesta de álcool nenhum dos pacientes teve óbito por cirrose hepática. O mecanismo que pode ser responsável por um aumento de tumores malignos neste grupo de pacientes parece ser o efeito sinérgico do tabagismo e abuso de álcool como fatores de risco ${ }^{7}$.

No caso relatado, o paciente persistiu com a ingesta frequente de bebidas alcoólicas por dez anos após o diagnostico da doença, a despeito da orientação médica. Além de aumentar o surgimento de depósitos lipomatosos podem ter contribuído para o surgimento do hepatocarcinoma.

O diagnóstico precoce é essencial para uma boa evolução clínica desses pacientes, através do esclarecimento dos riscos, orientações de perda de peso, abstinência alcoólica e rastreamento de neoplasias.

\begin{abstract}
The Madelung's disease, known as benign symmetric cervical lipomatosis is a rare condition of unknown etiology characterized by massive accumulation of fat mass. There are few studies on Madelung's disease and association with neoplasia. We describe a case of Madelung's disease associated with hepatocellular carcinoma.
\end{abstract}

Keywords: Madelung Disease. Adenomatous Lipomatosis. Alcoholism. Carcinoma, Hepatocellular.

\section{Referências}

1. Smith PD, Stadelmann WK, Wassermann RJ, Kearney RE. Benign symmetric lipomatosis (Madelung's disease). Ann Plast Surg. 1998; 41:671-3.

2. John DG, Fung HK, van Hasselt CA, King WW. Multiple symmetrical lipomatosis in the neck. Eur Arch Otorhinolaryngol. 1992; 249:277-8.
3. Bortoncello AC, Silva RV, Bortoncello RL, Daldon PEC. Doença de Madelung. Relato de caso. Rev Bras Clin Med 2010; 8: 362-4

4. Uhlin S. Benign Symmetric Lipomatosis. Arch Dermatol Jan 1979; 115:94-95.

5. Enzi G, Busetto L, Ceschin E, Coin A, Digito M, Pigozzo S. Multiple symmetric lipomatosis: clinical aspects and outcome in a long-term longitudinal study. Int J Obes Relat Metab Disord. 2002;26:253-61. 
6. Nielsen S, Levine J, Clay R, Jensen MD. Adipose Tissue Metabolism in Benign Symmetric Lipomatosis. J Clinical Endocrinol ans Metabolism March 2001; 86:2717-20

7. Enzi G, Busetto L, Ceschin E, Coin A, Digito M, Pigozzo S. Multiple symmetric lipomatosis: clinical aspects and outcome in a long-term longitudinal study. Int J Obes Relat Metab Disord. 2002; 26:253-61.

8. Agaimy A, Ihrler S, Märkl B, Lell M, Zenk J, Hartmann A, Michal M, Skalova A. Lipomatous salivary gland tumors: a series of 31 cases spanning their morphologic spectrum with emphasis on sialolipoma and oncocytic lipoadenoma. Am J Surg Pathol. 2013 Jan;37:128-37.
9. Linares Torres P, Castañón López C, Llano Alonso C, Alvarez Posadilla M, Vivas Alegre S, Espinel Díez J, et al. Association of adenocarcinoma of esophagus and breast cancer in a male with Madelung' disease. An Med Interna. 2006; 23:1335.

10. Borriello M, Lucidi A, Carbone A, lannone V, Ferrandina G. Malignant transformation of Madelung's disease in a patient with a coincidental diagnosis of breast cancer: a case report. Diagn Pathol. 2012;1:7:116. 